\title{
Robotic Kidney and Spine Percutaneous Procedures Using a New Laser-Based CT Registration Method
}

\author{
Alexandru Patriciu ${ }^{1,2}$, Stephen Solomon MD ${ }^{1,3}$, Louis Kavoussi MD ${ }^{1}$, \\ Dan Stoianovici $\mathrm{PhD}^{1,2}$ \\ ${ }^{1}$ Johns Hopkins Medical Institutions, Brady Urology Institute, URobotics Laboratory \\ \{patriciu, kavoussi, dss\} @urology.jhu.edu \\ ${ }^{2}$ Johns Hopkins University, Mechanical Engineering Department \\ ${ }^{3}$ Johns Hopkins Medical Institutions, Department of Radiology
}

\begin{abstract}
We present a simple method for robot registration in computer tomography imaging systems. The method uses the laser markers readily available on any CT scanner and does not require imaging thus eliminating radiation exposure. Its accuracy is inherited from the laser positioning system. This approach does not require additional hardware, laser alignment being performed on the instrument used in the clinical application. Moreover, robotic guidance allows for radiological interventions to be performed on scanners without fluoro-CT capability. Unlike the manual approach, the method allows for performing oblique insertions, for which the skin entry point and the target are located in different slices.

The implementation is realized using the latest version of the PAKY-RCM robot developed in our laboratory. This is an increased precision system based on our new Ball-Worm technology.

The system was successfully used for five CT-guided biopsy and radiofrequency ablation procedures on the kidney and spine and a nephrostomy tube placement. Further investigation will explore its application to other organs and procedures.
\end{abstract}

\section{Introduction}

Computer tomography (CT) guided percutaneous procedures are becoming increasingly popular in radiological interventions. CT guided interventions have been facilitated by the development of the CT fluoroscopy (CTF) imaging systems [5]. This new generation of CT-scanners allows for fluoro-imaging of a CT slice. Using the real-time cross-section image the radiologist manually orients and inserts a procedure needle towards the target, provided that the skin entry point and the target are located in the current fluoro slice. Even though the procedure is fast and precise in experienced hands, the major limitation of CTF is the relatively high radiation exposure to patient and physician $[6,15]$. In order to make the real time adjustments in needle trajectory the physician's hand is in or near the scanning plane. Physician hand exposure has been theoretically and empirically determined to be approximately $2 \mathrm{mGy}$ per procedure [11]. Kato et al. [8] have calculated that on the basis of an annual dose limit of $500 \mathrm{mSv}$ for the hands, a physician would be limited to performing only four CTF procedures per year. 
A number of procedural techniques, shields [12], and passive needle holders [1] have been proposed to reduce radiation exposure. Robotic systems have been investigated for eliminating radiation exposure and simultaneously increasing accuracy in radiological interventions 17,19. A system using CT-fluoroscopy was reported by Loser and Navab 9 . This uses a visual-servoing algorithm to orient the procedure needle based on fluoro-CT images. The approach demonstrated good targeting accuracy by using the procedure needle as a marker, without additional registration hardware. Even though the radiation exposure of the surgeon, which supervises the procedure from the control room, is virtually zero the patient is being exposed to radiation during the robot's image-based servo orientation.

Susil et al. [20 reported a registration method using a localization device (a modified Brown-Roberts-Wells frame $|1|$ ) attached to the robot's end-effector, which was further perfected by Masamune |10|. The method presents the advantage of providing the registration data from a single image slice. In addition the method is not restricted to the use of CTF. In our clinical experience implementing their method 13., however, the registration frame was cumbersome in the confined gantry space, and its initial positioning with respect to the CT active field imposed stringent constraints for interventional use.

The proposed method is significantly different from the above two in that it is not an image-based registration method. Similar to the Navab method, it requires no additional hardware and, alike the Susil system, it is not limited to the use of CTF scanners. Its laser-based registration principle insures zero radiation exposure for both the patient and personnel.

\section{Methods}

The system comprises a CT scanner, a personal computer (PC), and the PAKY-RCM robot $[3,14,16,17]$ attached to the $\mathrm{CT}$ table. The $\mathrm{PC}$ is equipped with a motion control card for robot control and acquires CT images in DICOM format through a network connection. The laser markers commonly available on the CT scanner are used for robot registration through a needle alignment processes. The radiologist chooses the target in the slice image displayed on the PC monitor, and the robot automatically aligns and delivers the needle. Several software and hardware mechanisms insure the safety of the procedure.

\subsection{The Robotic System}

The main component of the system is the PAKY - RCM robot. This robot has two components the PAKY (Percutaneous Access of the Kidney) needle driver and the RCM ( $\underline{\text { Remote }}$ Center of $\underline{\text { Motion) }}$ robot.

PAKY is a radiolucent needle driver used to guide and actively drive a trocar needle in X-Ray guided percutaneous access procedures. The needle driver is radiolucent thus allowing unobstructed visualization of the anatomical target and radiological guidance of the needle 16 . An electric motor performs automated needle insertion. PAKY has been successfully used in numerous clinical cases [3. 
The RCM robot is a compact robot for surgical applications that implements a fulcrum point located distal to the mechanism 17. The robot presents a compact design: it may be folded into a $171 \times 69 \times 52 \mathrm{~mm}$ box and it weighs only $1.4 \mathrm{Kg}$. The robot can precisely orient a surgical instrument in space while maintaining the location of one of its points. This kinematic architecture makes it proper for minimally invasive applications as well as trocar/needle orientation in percutaneous procedures. RCM accommodates various end-effectors. The robot was successfully used at the Johns Hopkins Medical Institutions for numerous surgical procedures [1]. The latest version of the RCM robot includes the Ball-Worm Transmission |18 developed in our laboratory and redundant encoding. These significantly enhance the safety, kinematic performance, and rigidity of the mechanism.

In the current setting the PAKY-RCM robot is used to orient a needle while maintaining its initial tip location and perform the insertion of the needle. Two degrees of freedom (DOF) are used for needle alignment and one translational DOF is used for needle insertion. For safety, the orientation and insertion stages may be independently enabled / disabled by hardware means.

The robotic assembly is fixed into a passive arm. This is mounted on a bridge fixture attached over the CT table. The passive arm allows for the support of the mechanism in close proximity of the targeted organ so that the tip of the needle is located at the desired skin entry point. In this setting only two rotations and one translation are required for accessing any nearby target.

\subsection{CT-Robot Laser Registration}

CT scanners are normally equipped with three laser markers as schematically presented in Figure 1 The Laser Plane 1 (LP1) coincides with the current CT image plane. The Laser Plane 2 (LP2) is parallel with LP1 and positioned at the distance $-\mathrm{Z}_{12}$

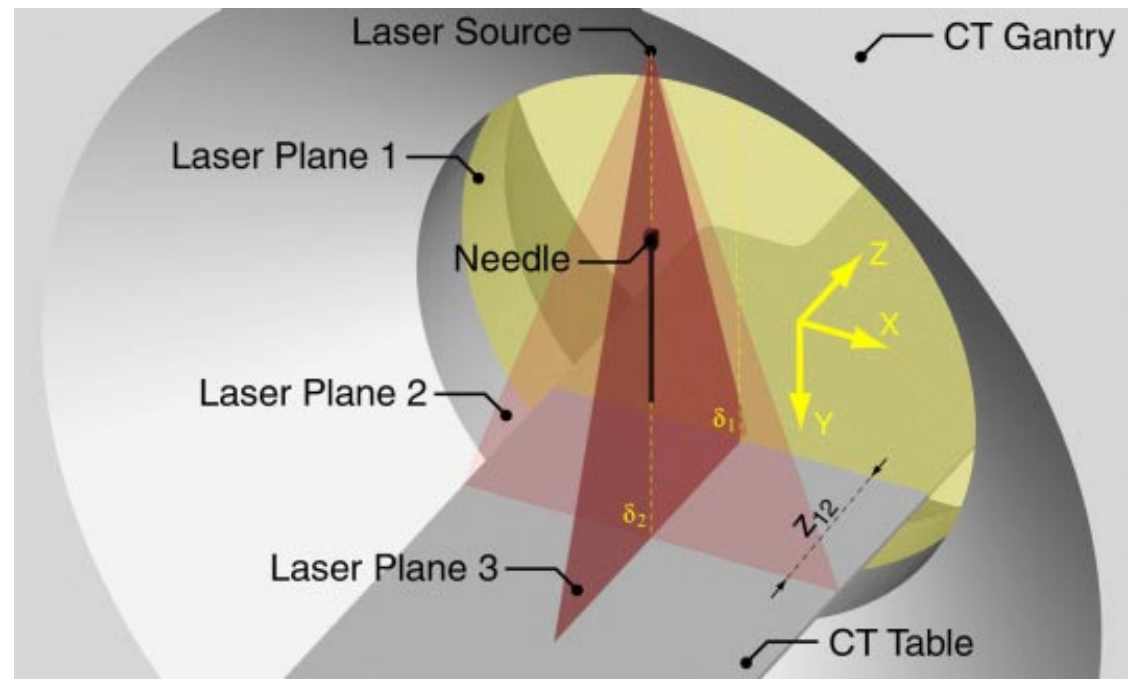

Figure 1: CT Laser Markers and Laser Registration Principle 
along the Z-axis of the CT scanner. The Laser Plane 3 (LP3) is perpendicular on LP1 and LP2 and defines the YOZ plane of the CT. The intersection of the LP1 and LP3 defines the vertical direction $\delta_{2}$ in the CT image space.

The proposed registration method is based on the alignment of the instrument (needle) with the vertical direction $\delta_{2}$. This is achieved by simultaneously aligning the needle in the LP2 and LP3 laser planes. The central and vertical direction of the current CT image $\delta_{1}$ is then obtained by a simple $\mathrm{z}_{12}$ translation. This alignment with the laser planes provides a 5 DOF registration. The remaining DOF, specifically the $Y$ position of the needle tip is unknown and remains to be determined from the CT image acquired for target specification. Alternatively, a forth laser could be used for providing 6 DOF laser registration by marking a horizontal plane. CT scanners, however, are not normally instrumented with this $4^{\text {th }}$ laser marker. Subsets of this methodology may also be implemented for particular applications and robot kinematic schemes requiring reduced DOF registration.

In our initial implementation we used a combined Laser-Image Registration method. This was required by the limited mobility structure of the PAKY-RCM robot used. The registration procedure involves two main steps, as follows:

Step 1: This defines the current image plane (LP1) in the robot coordinate system by using the laser alignment process. The robot is attached to the mobile CT table through a bridge mount and a passive positioning arm. Using the passive arm, the robot is placed so that the tip of the needle is located at the desired skin entry point. The CT table, together with the robot, is then moved until the tip of the needle is highlighted by the LP1 laser (LP2 and a translation could also be used). Figure 2 presents a schematic of several consecutive needle positions $\mathrm{P}_{1} \mathrm{O}, \mathrm{P}_{2} \mathrm{O}, \mathrm{P}_{3} \mathrm{O}, \mathrm{P}_{4} \mathrm{O}$ with the needle point $\mathrm{O}$ located in the image and laser plane LP1.

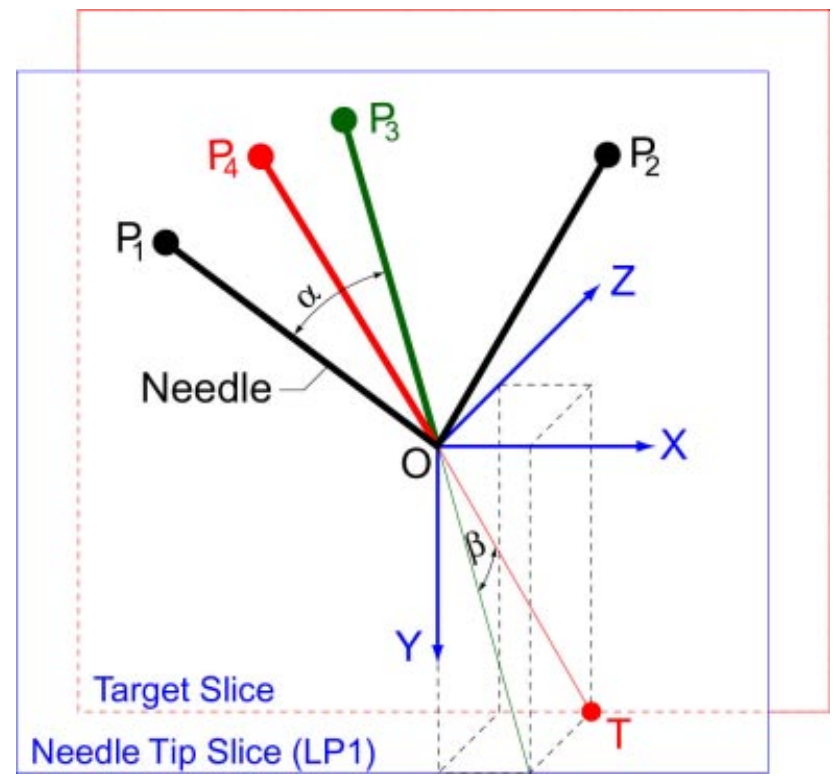

Figure 2: Laser - Image Registration Scheme 
The robot is moved under joystick control while observing the laser projection on the needle so that its head is aligned with the laser. During this motion, the RCM robot insures that the needle tip remains in LP1. In the $\mathrm{P}_{1} \mathrm{O}$ position of the needle the laser shines its entire barrel. In our approach this was set and inspected by direct observation. For providing automated positioning, a future approach will use a laser detection sensor mounted at the needle head. As such, the needle $\mathrm{P}_{1} \mathrm{O}$ is located in the current image and laser plane LP1. The PC acquires this needle orientation by recording the robot joint coordinates.

The process of needle alignment in the laser plane LP1 is then repeated for a dissimilar orientation of the needle $\mathrm{P}_{2} \mathrm{O}$. Joint coordinates are acquired at this position and geometric calculations are employed to define the $\mathrm{P}_{1} \mathrm{OP}_{2}$ plane in the robot coordinate system. This defines the current image plane in robot space, thus providing the first 2 DOF for the registration process. At this stage the robot may be restricted to move in the LP1 image plane. This could be used to remotely manipulate the needle in the image space, in a similar way that radiologists presently perform CTF manual interventions.

Step 2: The remaining registration data is image-based and uses the image acquired for entry-point / target specification. An image is acquired at the same $\mathrm{P}_{1} \mathrm{O}$ orientation of the needle. The combined registration data is then calculated by overlapping the needle $\mathrm{P}_{1} \mathrm{O}$ in the image and robot spaces, providing the complete registration data, which will be used for orienting the needle towards the specified target.

\subsection{Targeting Methodology}

An image slice is acquired through the needle-tip and one through the desired target (Needle Tip Slice and Target Slice in Figure 2. In the particular case that the needle tip and the target are located in the same image plane, only one acquisition is required. The images are acquired in DICOM format and displayed on the PC monitor. The radiologist identifies the target by using the mouse.

The transversal targeting angle $(\alpha)$ is determined by using simple geometric relations in the Target Slice image. The longitudinal targeting angle $\beta$ is then calculated by using the distance between the two slices retrieved from the DICOM images.

Under the control of the radiologist the robot automatically orients the needle at the position $\mathrm{P}_{4}$ specified by the angles $\alpha$ and $\beta$ through the intermediary (in plane) position $\mathrm{P}_{3}$ (given by $\alpha$ ). In the particular case that the target and skin entry point are located in the same slice, all calculations are performed on the same image and $\beta=0$. The needle depth of insertion is calculated by using the image of the target and needle tip in the two slices.

\section{Results}

Preliminary accuracy testing was performed in-vitro using $1 \mathrm{~mm}$ diameter metallic balls. The target was placed in the same image plane with the needle tip and also in different planes. The targeting error achieved over fifty experiments was less than 1 
$\mathrm{mm}$ in plane and $1.5 \mathrm{~mm}$ for out of plane targets. With these satisfactory results, the extensive clinical experience with the PAKY-RCM robot in percutaneous renal access under C-Arm guidance $[1,3,17]$, and the safety of the PAKY-RCM robot rendered by its decoupled needle orientation and insertion capability, we proceeded for the clinical application. For verifying needle-targeting accuracy before needle insertion, in our studies we used a CTF scanner (Siemens - Somatom Plus Four). In addition, in all clinical applications we performed several algorithm-testing steps insuring the functionality of the algorithm in successive phases.
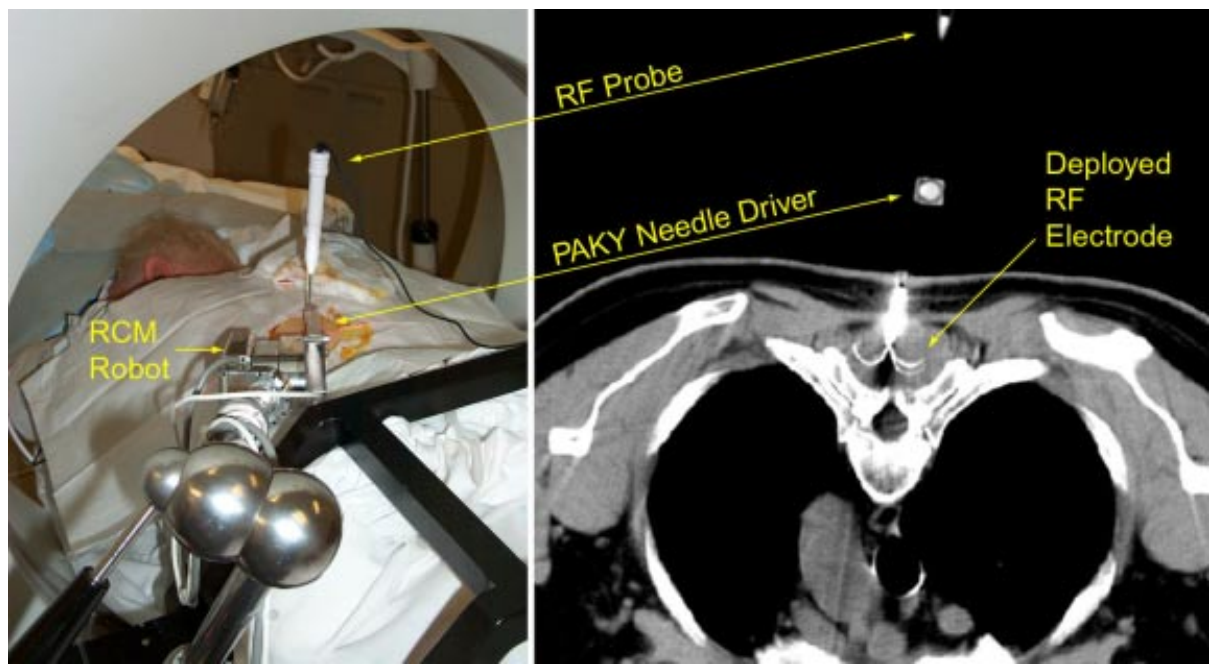

Figure 3: Robotic CT-Guided Spine Radio Frequency Ablation

Five robotic radiological interventions including a nephrostomy tube, kidney and spine biopsy and radio frequency ablation have been successfully performed (Figure 3, Figure 4), as follows:

- The patients were placed in the prone position on the CTF table. A volume scan was initially acquired to localize the lesion and plan the procedure. The patient's back was cleaned with betadine and local lidocaine was administered over the planned entry site. A small nick was made in the skin at the desired entry site.

- The mechanical arm with a sterilized PAKY needle driver holding the needle (Temno $18 \mathrm{~g} / 15 \mathrm{~cm}$ for biopsy and 3.0 Radio Therapeutics for ablation) was placed such that the tip of the needle was located at the skin incision site. The table was then moved, together with the robot, so that the needle tip was located in the laser plane.

- Using the joystick the robot was moved in two different position $\left(\mathrm{P}_{1}\right.$ and $\left.\mathrm{P}_{2}\right)$ located in the laser plane, these directions were acquired by the $\mathrm{PC}$, and used for computing the position of the CT-slice in the robot space. For testing, the control was then transferred to the computer and the robot was moved back and forth in the laser plane to insure its correct determination. This was visually acknowledged by observing the laser projection on the barrel of the needle during 
the in-plane motion. In all needle orientation phases the tip of the needle was located at the skin site and needle insertion was hardware disabled.

- A scan was that taken with the needle at the first position $\left(\mathrm{P}_{1}\right)$. The image was transferred to the $\mathrm{PC}$, the needle tip was identified in the image, and the orientation of the needle in image was determined, finalizing the registration process. To verify the registration result, the needle was moved to the vertical inslice position and a new image slice was acquired for confirmation.

- A second scan was acquired through the targeted lesion. This image was also transferred to the PC and the radiologist indicated the lesion on the PC monitor. The program computed the needle targeting angles $(\alpha$ and $\beta)$ and the required depth of insertion.

- With needle insertion disabled, under the command of the radiologist the robot oriented the needle towards the target. During this motion the needle was entirely outside the body only its tip being located at the skin level. Needle orientation accuracy was than verified by the radiologist under fluoro imaging.

- Finally, the RCM orientation stage was disabled and the PAKY needle driver was enabled on the hardware. At the radiologist's command the needle was inserted under direct fluoro supervision.

- The remaining steps of the procedure were then performed as usual.

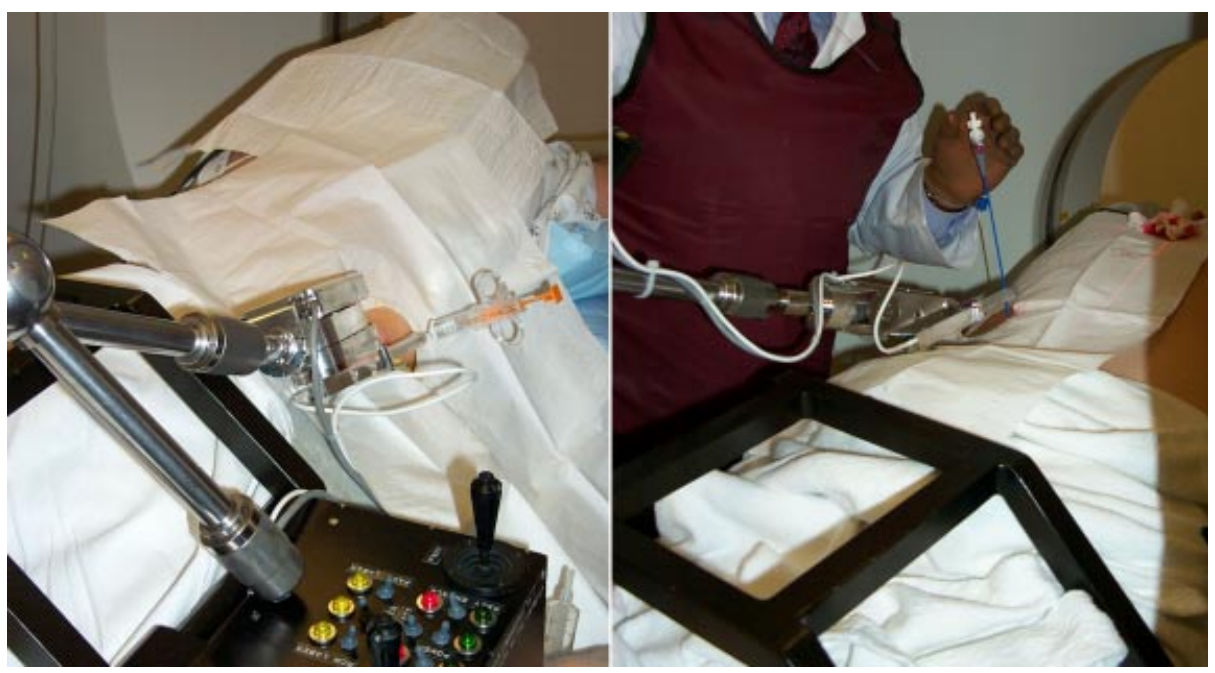

Figure 4: Robotic CT-Guided Kidney Biopsy and Nephrostomy Tube Procedures

The methodology presented above includes numerous verification/confirmation steps that will be eliminated after a complete evaluation of the methodology. This would significantly shorten and speed up the clinical process. The following table summarizes the radiological interventions performed using the above methodology: 
Table 1: Robotic CT-Guided Interventions

\begin{tabular}{|l|l|l|l|}
\hline Case No. & Date & Organ & Procedure \\
\hline 1 & February 06, 2001 & Kidney & Biopsy \\
\hline 2 & February 15, 2001 & Kidney & Nephrostomy Tube \\
\hline 3 & March 08, 2001 & Kidney & Biopsy and RF Ablation \\
\hline 4 & March 08, 2001 & Spine & RF Ablation \\
\hline 5 & March 08, 2001 & Spine & RF Ablation \\
\hline
\end{tabular}

\section{Conclusions}

We presented a simple CT-robot registration method using the laser markers of the CT scanner and its application to CT-guided interventional procedures. The registration may be used with traditional (non CTF) scanners and it does not involve additional registration devices, using the procedure instrument (needle) as a registration marker. The method allows for performing needle access in an oblique direction, for which the skin entry point and the target are located in different CT slices. This is a significant improvement over the manual method, in which the needle is restricted to the fluoro image of the CTF scanner. The accuracy of the in-vitro experiments and successful clinical cases performed demonstrated the effectiveness of the method. The method's accuracy is inherited from the laser positioning system of the CT scanner. Its clinical implementation on the new PAKY-RCM robot proved safe and reliable in various radiological interventions. It provides null radiation exposure for the radiologist controlling the procedure from the control room and minimizes the exposure of the patient. With the use of the proposed system the radiation exposure does not bound the number of CT interventions a radiologist may safely perform. Moreover, through the use of a specialized algorithm and precise delivery mechanism, the method reduces physician's variability in performing CTguided percutaneous access. Future applications of this technology to other radiological interventions are in progress.

\section{References}

1. Bishoff JT, Stoianovici D, Lee BR, Bauer J, Taylor RH, Whitcomb LL, Cadeddu JA, Chan D, Kavoussi LR: RCM-PAKY: Clinical Application of a New Robotic System for Precise Needle Placement, (1998), Journal of Endourology, 12:82

2. Brown RA, Roberts TS, Osborne AG: Stereotaxic frame and computer software for CT directed Neurosurgical localization. Invest. Radiol. (1980), 15: 308-312

3. Cadeddu JA, Stoianovici D, Chen RN, Moore RG, Kavoussi LR: Stereotactic mechanical percutaneous renal access, (1998), Journal of Endourology, 12:2:121-126.

4. Daly B, Krebs TL, Wong-You-Cheong JJ, Wang SS: Percutaneous abdominal and pelvic interventional procedures using ct fluoroscopy guidance. AJR (1999) 173:637-644

5. Gianfelice D, Lepanto L, Perreault P, Chartrand-Lefebvre C, Milette PC: Value of ct fluoroscopy for percutaneous biopsy procedures. JVIR 2000 (2000) 11:879-884

6. Gianfelice D, Lepanto L, Perreault P, Chartrand-Lefebvre C, Milette PC: Effect of the learning process on procedure times and radiation exposure for ct fluoroscopy-guided percutaneous biopsy procedures. JVIR (2000) 11:1217-1221 
7. Glauser D: Neurosurgical robot Minerva, first results and current developments. Proc. Sec. Int. Symp. On Med Rob and Computed Assisted Surgery (1995).

8. Kato R, Katada K, Anno H, Suzuki S, Ida Y, Koga S.: Radiation dosimetry at CT fluoroscopy: physician's hand dose and development of needle holders. Radiology (1996) 201:576-578

9. Loser $\mathrm{MH}$, Navab $\mathrm{N}$ : A new robotic system for visually controlled percutaneous interventions under CT fluoroscopy, MICCAI 1999, Lecture Notes in Computer Science, Springer-Verlag (2000) 1935:887-896

10. Masamune K, Patriciu A, Stoianovici D, Susil R, Taylor RH, Fichtinger G, Kavoussi LR, Anderson J, Sakuma I, Dohi T: Development of CT-PAKY frame system - CT image guided Needle puncturing manipulator and a single slice registration for urological surgery, Proc. 8th annual meeting of JSCAS, Kyoto 1999:89-90

11. Nawfel RD, Judy PF, Silverman SG, Hooton S, Tuncali K, Adams DF: Patient and personnel exposure during ct fluoroscopy-guided interventional procedures. Radiology (2000) 216:180-184

12. Nickoloff EL, Khandji A, Dutta A: Radiation doses during ct fluoroscopy. Health Physics (2000) 79:675-681

13. Patriciu A, Mazilu D, Stoianovici D, Stanimir A, Susil R, Masamune K, Fitchinger G, Taylor RH, Anderson J, Kavoussi LR: CT-Guided Robotic Prostate Biopsy, (2000), 18th World Congress on Endourology \& SWL, Sept. 2000, Sao Paulo, Brazil.

14. Patriciu A, Stoianovici D, Whitcomb LL, Jarrett T, Mazilu D, Stanimir A, Iordachita I, Anderson J, Taylor R, Kavoussi LR: Motion-Based Robotic Instrument Targeting Under C-Arm Fluoroscopy, (2000), MICCAI, Lecture Notes in Computer Science, SpringerVerlag, 1935:988-998.

15. Silverman SG, Tuncali K, Adams DF, Nawfel RD, Zou KH, Judy PF: CT fluoroscopyguided abdominal interventions: techniques, results, and radiation exposure. Radiology (1999) 212:673-681

16. Stoianovici D, Cadeddu JA, Demaree RD, Basile HA, Taylor RH, Whitcomb LL, Sharpe W, Kavoussi LR: An Efficient Needle Injection Technique and Radiological Guidance Method for Percutaneous Procedures, (1997), Lecture Notes in Computer Science, Springer-Verlag, 1205:295-298

17. Stoianovici D, Whitcomb LL, Anderson JH, Taylor RH, Kavoussi LR: A Modular Surgical Robotic System for Image Guided Percutaneous Procedures, (1998) Lecture Notes in Computer Science, Springer-Verlag, 1496:404-410

18. Stoianovici D, Kavoussi LR: Ball-Worm Transmission, (1999), Regular U.S. utility and PCT application filled by the Johns Hopkins University (\#DM-3512)

19. Stoianovici D: Robotic Surgery, (2000) World Journal of Urology, 18:4:289-295. (http://link.springer.de/link/service/journals/00345/tocs/t0018004.htm)

20. Susil RC, Anderson J, Taylor RH: A Single Image Registration Method for CT Guided Interventions. MICCAI 1999, Lecture Notes in Computer Science, Springer-Verlag (1999) 1679:798-808 